\title{
Repeated magnetic resonance imaging and Cyberknife therapy for Parkinson's disease in a patient with a pacemaker and epicardial leads
}

\author{
Andrzej Kułach, Tomasz Skowerski, Zbigniew Gąsior
}

Department of Cardiology, School of Health Sciences, Medical University of Silesia, Katowice, Poland

Submitted: 9 March 2019

Accepted: 5 May 2019

Arch Med Sci 2020; 16 (6): 1470-1471

DOI: https://doi.org/10.5114/aoms.2020.100308

Copyright $\odot 2020$ Termedia \& Banach

Patients with pacemakers are commonly advised that they cannot undergo magnetic resonance imaging (MRI) scanning, even though it is possible and safe to scan the majority of pacemakers. Epicardial leads, however, are considered absolute contraindication to magnetic resonance imaging [1].

We report a case of a 68-year-old male patient with a history of atrioventricular (AV) second degree block who had a dual chamber pacemaker (Medtronic Sensia) with endocardial leads implanted in 2009. Two months later due to an exit block, he was admitted for right ventricular (RV) lead replacement. Then, due to thrombosis of the left brachiocephalic vein, the endocardial leads were removed and the patient had epicardial leads implanted (Medtronic CapSure Epi 4968, steroid-eluting bipolar leads) with a pacemaker placed in the abdominal wall. Since then the device parameters had been stable.

In 2014 the patient was diagnosed with Parkinson's disease. In May 2017, because of therapy resistance he was qualified for Cyberknife therapy, and MRI had to be performed to plan the procedure. Due to severe neurological symptoms, despite contraindications, we decided to proceed with an MRI (GE Optima). We did not observe any complications during/after MRI. The device parameters remained unchanged (Table I).

In April 2018 the pacemaker was replaced with an Ensura MRI Surescan system because of the end of battery life. The epicardial leads were left intact. A few months after Cyberknife the Parkinson's disease symptoms relapsed and another MRI was suggested to plan deep brain stimulation. In August 2018 another MRI was performed. The device parameters also remained unchanged. During both $M R I$ studies a cardiologist, radiologist and MRI physicist were present. The patient was pacemaker dependent (VP 95-98\%, escape rhythm < $40 \mathrm{bpm}$ ). The pacemaker was programmed to DOO mode $90 \mathrm{bpm}$ with all anti-tachycardia functions switched off and reprogrammed to baseline settings after the study. Heart rate and oxygen saturation were monitored continuously with a pulse oximeter. Study parameters, including magnetic field, sequences and specific absorption rate (SAR), are reported in Table I.

Epicardial leads are rarely used in adult patients these days and are considered in case of infective complications or when vascular access problems occur (e.g. venous thrombosis). They are thought to be less durable, more prone to malfunction and have higher thresholds.

\author{
Corresponding author: \\ Tomasz Skowerski MD \\ Department of Cardiology \\ School of \\ Health Sciences \\ Medical University \\ of Silesia \\ 45 Ziołowa St \\ 40-635 Katowice, Poland \\ Phone: +48 607234440 \\ E-mail: \\ tskowerski@gmail.com
}


Table I. Pacemaker parameters before and after MRI study

\begin{tabular}{|c|c|c|c|c|c|c|}
\hline Variable & $\begin{array}{c}\text { Atrial } \\
\text { impedance } \\
{[\Omega]}\end{array}$ & $\begin{array}{c}\text { Ventricular } \\
\text { impedance } \\
{[\Omega]}\end{array}$ & $\begin{array}{l}\text { Atrial capture } \\
\text { threshold } \\
\text { [V@ms] }\end{array}$ & $\begin{array}{c}\text { Ventricular } \\
\text { capture } \\
\text { threshold } \\
\text { [V@ms] }\end{array}$ & $\begin{array}{l}\text { Atrial } \\
\text { sensing } \\
{[\mathrm{mV}]}\end{array}$ & $\begin{array}{c}\text { Ventricular } \\
\text { sensing } \\
{[\mathrm{mV}]}\end{array}$ \\
\hline Before $1^{\text {st }} M R I$ & 537 & 738 & 0.875@0.4 & 1.3@0.4 & $>5.6$ & $>15$ \\
\hline After $1^{\text {st }} M R I$ & 522 & 722 & $0.75 @ 0.4$ & $1.0 @ 0.5$ & $0.7-1$ & $15-17$ \\
\hline $1^{\text {st }} \mathrm{MRI}$ - parameters & \multicolumn{6}{|c|}{$\begin{array}{l}\text { Static field strength: } 1.5 \text { T, spatial gradient: } 12,4 \mathrm{~T} / \mathrm{m} \text {, sequences: } \mathrm{T} 1, \mathrm{~T} 2 \text {, FLAIR, DWI, SWI; } \\
\text { SAR } 1.332 \mathrm{~W} / \mathrm{kg}\end{array}$} \\
\hline Before $2^{\text {nd }} M R I$ & 515 & 790 & $0.5 @ 0.4$ & $1.0 @ 0.4$ & $0.7-1$ & $>15$ \\
\hline After $2^{\text {nd }} M R I$ & 506 & 652 & $0.75 @ 0.4$ & 1.25@0.4 & $0.7-2.8$ & $>15$ \\
\hline $2^{\text {nd }} \mathrm{MRI}$ - parameters & \multicolumn{6}{|c|}{$\begin{array}{l}\text { Static field strenght: } 1.5 \mathrm{~T} \text {, spatial gradient: } 12,4 \mathrm{~T} / \mathrm{m} \text {, sequences: } \mathrm{T} 1, \mathrm{~T} 2 \text {, FLAIR, DWI, SWI; } \\
\text { SAR } 1.162 \mathrm{~W} / \mathrm{kg}\end{array}$} \\
\hline
\end{tabular}

In pediatric patients with congenital heart diseases epicardial leads are more commonly used. In a small case series $(n=11)$ Pulver et al. reported successful MRI in patients with congenital heart disease and implanted pacemakers. No inappropriate pacing or significant changes in parameters were noted [2]. Similarly, Hwang et al. reported a series of 4 patients with a cardiovascular implantable electronic device (CIED) and epicardial leads, who underwent MRI with no complications [3].

The 2017 Heart Rhythm Society Statement on $M R I$ radiotherapy in CIED patients upholds epicardial lead as a contraindication to MRI, but this is due to insufficient evidence to issue safety recommendation [4]. According to data obtained from the manufacturer, epicardial lead structure is not significantly different from the MR conditional endocardial lead. The major concern regarding the epicardial lead in the MR environment is possible excessive heating of pacing buttons (and subsequently increased pacing threshold), which may be explained by the lack of blood flow [5]. In vitro data suggest that the standard 1.5 T MR protocol generates negligible heating of leads not surrounded by blood.

In conclusion, our patient had MR scans performed twice. Based on our observation and literature data it seems that epicardial leads should not be considered an absolute contraindication and MR should be performed in such patients, whenever this modality is best to warrant proper diagnosis and treatment. The problem, however, calls for a larger set of data to address this matter.

\section{Conflict of interest}

The authors declare no conflict of interest.

\section{References}

1. Brignole M, Auricchio A, Baron-Esquivias G, et al. 2013 ESC guidelines on cardiac pacing and cardiac resynchronization therapy: the taskforce on cardiac pacing and resynchronization therapy of the European Society of Cardiology (ESC). Developed in collaboration with the European Heart Rhythm Association (EHRA). European Society of Cardiology (ESC). Europace 2013; 15: 1070-118.

2. Pulver AF, Puchalski MD, Bradley DJ, et al. Safety and imaging quality of MRI in pediatric and adult congenital heart disease patients with pacemakers. Pacing Clin Electrophysiol 2009; 32: 450-6.

3. Hwang YM, Kim J, Lee JH, et al. cardiac implantable electronic device safety during magnetic resonance imaging. Korean Circ J 2016; 46: 804-10.

4. Indik JH, Gimbel JR, Abe H, et al. 2017 HRS expert consensus statement on magnetic resonance imaging and radiation exposure in patients with cardiovascular implantable electronic devices. Heart Rhythm 2017; 14: e97-153.

5. Nazarian S, Roguin A, Zviman MM, et al. Clinical utility and safety of a protocol for noncardiac and cardiac magnetic resonance imaging of patients with permanent pacemakers and implantable-cardioverter defibrillators at 1.5 tesla. Circulation 2006; 114: 1277-84. 